\title{
Perivascular space is associated with brain atrophy in patients with multiple sclerosis
}

\author{
Xue-Yu Liu, Gai-Ying Ma, Shi Wang, Qian Gao, Cong Guo, Qiao Wei, Xuan Zhou, Li-Ping Chen \\ Department of Neurology, Key Laboratory of Neurology of Hebei Province, The Second Hospital of Hebei Medical University, Shijiazhuang, \\ China
}

Contributions: (I) Conception and design: XY Liu; (II) Administrative support: LP Chen; (III) Provision of study materials or patients: XY Liu, GY Ma; (IV) Collection and assembly of data: XY Liu, GY Ma, S Wang, Q Gao, C Guo; (V) Data analysis and interpretation: XY Liu, S Wang; (VI) Manuscript writing: All authors; (VII) Final approval of manuscript: All authors.

Correspondence to: Li-Ping Chen, MD. Department of Neurology, Key Laboratory of Neurology of Hebei Province, The Second Hospital of Hebei Medical University, No. 215 of Heping West Road, Xinhua District, Shijiazhuang 050000, China. Email: chenliping_dr@163.com.

Background: Perivascular space (PVS) is associated with neurodegenerative and neuroimmune diseases. Multiple sclerosis (MS) is traditionally a neuroimmune disease. However, studies show neurodegeneration also plays a vital role in MS. At present, most studies conclude severer PVS in MS is an imaging marker of neuroinflammation, while a 7T MRI study suggests that PVS in MS is associated with neurodegeneration.

Methods: In this study, 82 MS patients $(n=82)$ and 32 healthy controls $(n=32)$ were enrolled. The following indexes were measured: the number, size and distribution of PVS, the PVS score, corpus callosum index (CCI), corpus callosum area (CCA), the ratio of the corpus callosum to the cranium (CCR), aligned third ventricle width $(\mathrm{a} 3 \mathrm{VW})$, and unaligned third ventricle width $(\mathrm{u} 3 \mathrm{VW})$.

Results: The PVS score (4 vs. 3, $\mathrm{P}=0.041)$, PVSs number $(103.280 \pm 45.107$ vs. 87.625 $\pm 30.139, \mathrm{P}=0.035)$, and enlarged perivascular spaces (EPVSs) number ( 9 vs. $1, \mathrm{P}<0.001)$ of MS patients were significantly higher than in the healthy controls. PVSs number (23.5 vs. 13) and EPVSs number (1 vs. 0) in the basal ganglia (BG), and EPVSs number (3 vs. 0) in centrum semiovale (CSO) of MS patients were significantly higher than in the healthy controls, $\mathrm{P}<0.001$. In MS patients, PVS was correlated with age and hypertension but not to the extended disability status scale (EDSS) score and other clinical data. In MS patients, PVS score was correlated with CCA ( $\mathrm{rs}=0.272 ; \mathrm{P}=0.013$ ) and the CCR ( $\mathrm{rs}=0.219 ; \mathrm{P}=0.048$ ), and PVSs number was correlated with CCA ( $\mathrm{rs}=0.255 ; \mathrm{P}=0.021)$, the correlation disappeared after adjusting hypertension and age. In MS patients in remission, PVSs number was correlated with CCA ( $r s=0.487$; P=0.019), CCR ( $r s=0.479$; $\mathrm{P}=0.021)$, and PVS score was correlated with CCA ( $\mathrm{rs}=0.453 ; \mathrm{P}=0.03)$. After adjustment of hypertension and age, the total number of PVSs was correlated with CCA ( $\mathrm{rs}=0.419 ; \mathrm{P}=0.049$ ).

Conclusions: The PVS load in MS patients was heavier than healthy people, especially in BG and CSO. PVS was not correlated with EDSS in MS patients. The PVS of MS patients was associated with CCA and CCR, and PVSs number was independently related with CCA in MS patients in remission.

Keywords: Perivascular space (PVS); brain atrophy; multiple sclerosis (MS); neuroinflammation; neurodegeneration

Submitted Jul 07, 2021. Accepted for publication Oct 20, 2021.

doi: $10.21037 /$ qims-21-705

View this article at: https://dx.doi.org/10.21037/qims-21-705 


\section{Introduction}

Perivascular spaces (PVS) refer to the tissue spaces formed by accompanying and wrapping the small arteries and veins through the brain parenchyma at the microvascular levels and are composed of single or double layers of pia mater. PVS is filled with interstitial fluid rather than cerebrospinal fluid. PVS is a kind of normal anatomy. Generally, it is not visible (1). PVS is part of the blood-brain barrier and plays an essential role in brain fluid drainage, material transportation, and immune regulation. It may be one of the most important pathways for soluble proteins and leukocytes to enter the central nervous system (CNS) (2).

At present, there are many studies on PVS and CNS diseases. Previous studies revealed that aging (3), hypertension (4), cerebrovascular disease, neurodegeneration (such as Alzheimer's disease, dementia, etc.) $(5,6)$, and neuroimmune inflammation [such as multiple sclerosis (MS), neuromyelitis optica, etc.] could all lead to the expansion of the PVS, thus enlarged perivascular spaces (EPVS) are formed, which can be displayed on magnetic resonance imaging (MRI).

MS is traditionally a chronic immune-mediated inflammatory demyelinating disease of the CNS. However, in past studies, it has been revealed that the occurrence and development of disability in MS patients are contradictory to the neuroinflammatory lesions shown by MRI. That is, there is "clinico-radiological paradox $(7,8)$ ". The disease also has prominent neurodegenerative features, and it has a stronger correlation with clinical disability, but the correlation of the disease with neurodegeneration and neuroinflammation is unclear (9). Brain atrophy is a quantifiable MRI neurodegenerative index of MS (10).

PVS is associated with both neurodegenerative diseases and neuroimmune diseases. However, MS is a disease with both neuroinflammation and neurodegeneration. At present, most clinical studies on PVS and MS believe that PVS in MS is only related to neuroinflammation (11-16). A 7T MRI study also suggests that the occurrence of PVS in MS is associated with neurodegeneration (17).

This study aims to analyze and verify the PVS image characteristics in MS and determine the correlation between PVS and neurodegeneration in MS.

We present the following article in accordance with the MDAR checklist (available at https://dx.doi.org/10.21037/ qims-21-705).

\section{Methods}

\section{Subjects}

In this study, we retrospectively collected and analyzed the data of 82 patients $(n=82)$ diagnosed with MS treated at the Second Hospital of Hebei Medical University from 2017 to 2020, and 32 healthy volunteers who received physical examinations at the hospital at the same time were enrolled as the control group $(\mathrm{n}=32)$. The MS groups consisted of 24 patients with an initial neurological event (INE), 47 relapsing-remitting (RR), 11 progressive MS patients. The study was conducted in accordance with the Declaration of Helsinki (as was revised in 2013). The study was approved by Ethics Committee of the Second Hospital of Hebei Medical University (No. 2021-R433) and informed consent was taken from all the patients.

MS patients met the following inclusion criteria: (I) patients aged 15-65 years old; (II) patients who met the 2017 edition of the McDonald MS diagnostic criteria and completed a 3T MRI examination. Exclusion criteria: (I) patients with neuromyelitis optica spectrum disorders (NMOSD), acute disseminated encephalomyelitis (ADEM), idiopathic myelitis (IM), and patients with other neurological histories; (II) patients with aphasia, deafness, and mental disorders that affected investigation; (III) patients with poor image quality.

The inclusion criteria of healthy volunteers were as follows: (I) subjects without a nervous system disease; (II) subjects without evidence of a pathological MRI; (III) subjects without a contraindication for an MRI examination.

\section{Clinical data acquisition}

Clinical information was collected from patients, including age, gender, hypertension, diabetes, hyperlipidemia, smoking and drinking history. The extended disability status scale (EDSS) was used to evaluate the physical condition of MS patients at the time of the MRI examination, where EDSS $<3$ indicates mild disability, and EDSS $\geq 3$ indicates severe disability.

\section{MRI scheme}

MRI images were collected using a 3.0 T superconducting MRI scanner produced by Philips equipped with an 

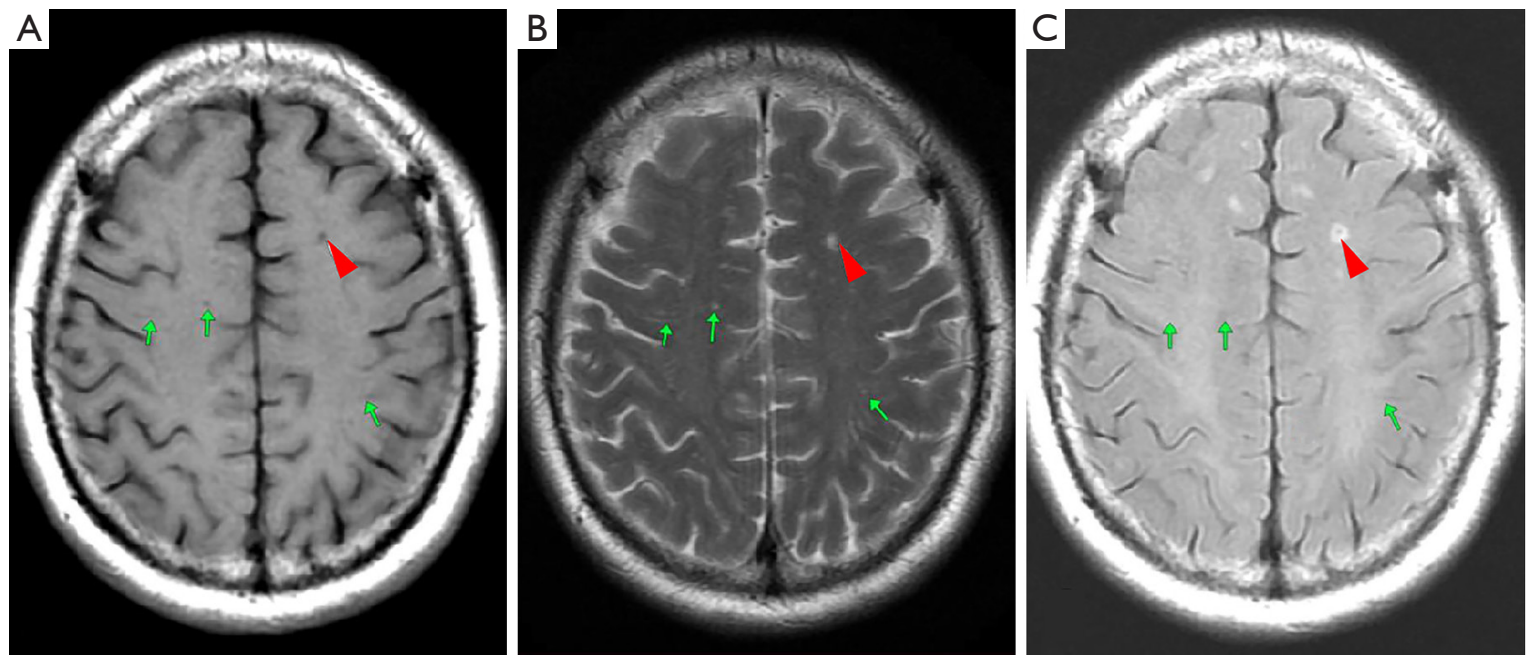

Figure 1 PVS on MRI. The green arrow indicates a typical PVS. The cavity pointed by the red triangle has a high signal edge, not PVS. PVS, perivascular space; MRI, magnetic resonance imaging.

8-channel head quadrature coil. Axial scanning with T1 weighted (T1WI), T2 weighted (T2WI), and T2-FLAIR sequences, and sagittal scanning with T1 weighted (T1WI) sequences were performed. Scan parameters: T1WI: repetition time (TR) $190 \mathrm{~ms}$, echo time (TE) $2 \mathrm{~ms}$; T2WI: TR 5,000 ms, TE $110 \mathrm{~ms}$; T2WI: FLAIR TR 7,000 ms, TE $140 \mathrm{~ms}$; the slice thickness was $6 \mathrm{~mm}$, the interslice gap was $5 \mathrm{~mm}$; and field of view (FOV) was $230 \mathrm{~mm} \times 230 \mathrm{~mm}$.

\section{MRI interpretation and analysis}

MRI images were interpreted and analyzed by two neuroimaging doctors blinded to the clinical information using the Radiant software.

\section{PVS interpretation and scoring}

PVS is generally defined as round, oval, or linear lacuna with clear edges on MRI, which is located in the area supplied by the perforating artery, with an isointensive cerebrospinal fluid (CSF) signal (hypointensive signal on T1, hyperintensive signal on T2, FLAIR isointensive signal but usually without a hyperintensive signal edge). Generally, the maximum diameter is $<3 \mathrm{~mm}$. For the lesions that meet the above criteria but whose maximum diameter was $\geq 3 \mathrm{~mm}$, PVS was diagnosed only when it had a typical vascular shape and followed the direction of the perforating artery. Because of the quality of the MRI image, interpretation was mainly based on the T2 image (example: PVS on MRI, Figure 1).
The number and distribution of PVS were recorded. When measuring the size of PVS, for circular PVS, the diameter was measured. For elliptic PVS, the short diameter was measured. For linear PVS, the maximum diameter of its minor axis was measured. PVS with a diameter $>2 \mathrm{~mm}$ was defined as EPVSs.

PVS was scored according to the literature published by Potter et al. (18). For the centrum semiovale (CSO) and basal ganglia (BG), each hemisphere was evaluated and scored separately. Then, when the hemispheres were asymmetric, the side with a higher score was scored. The PVS scores were 0 (none), 1 (1-10 PVSs), 2 (11-20 PVSs), 3 (21-40 PVSs), and 4 (>40 PVSs). According to the presence of PVS, the score was 0 (without PVS) or 1 (with PVS) for the midbrain and hippocampus. As PVS can be seen in the anterior commissure of healthy subjects and patients, the anterior commissure layer was not involved in the scoring. The final PVS score was the sum of the scores of the four regions. Theoretically, the PVS score may be 0-10 (example: PVS of different brain regions, see Figure 2).

\section{Measurement of brain atrophy}

Commonly used imaging evaluation methods of brain atrophy in clinic are as below: (I) linear measurement, which is easy to obtain and reliable; (II) area measurement that can indirectly reflect the brain volume by measuring the area of each part of the brain; (III) volume measurement, accurate but difficult to obtain. People could choose these methods according to their needs in clinic or in research. 

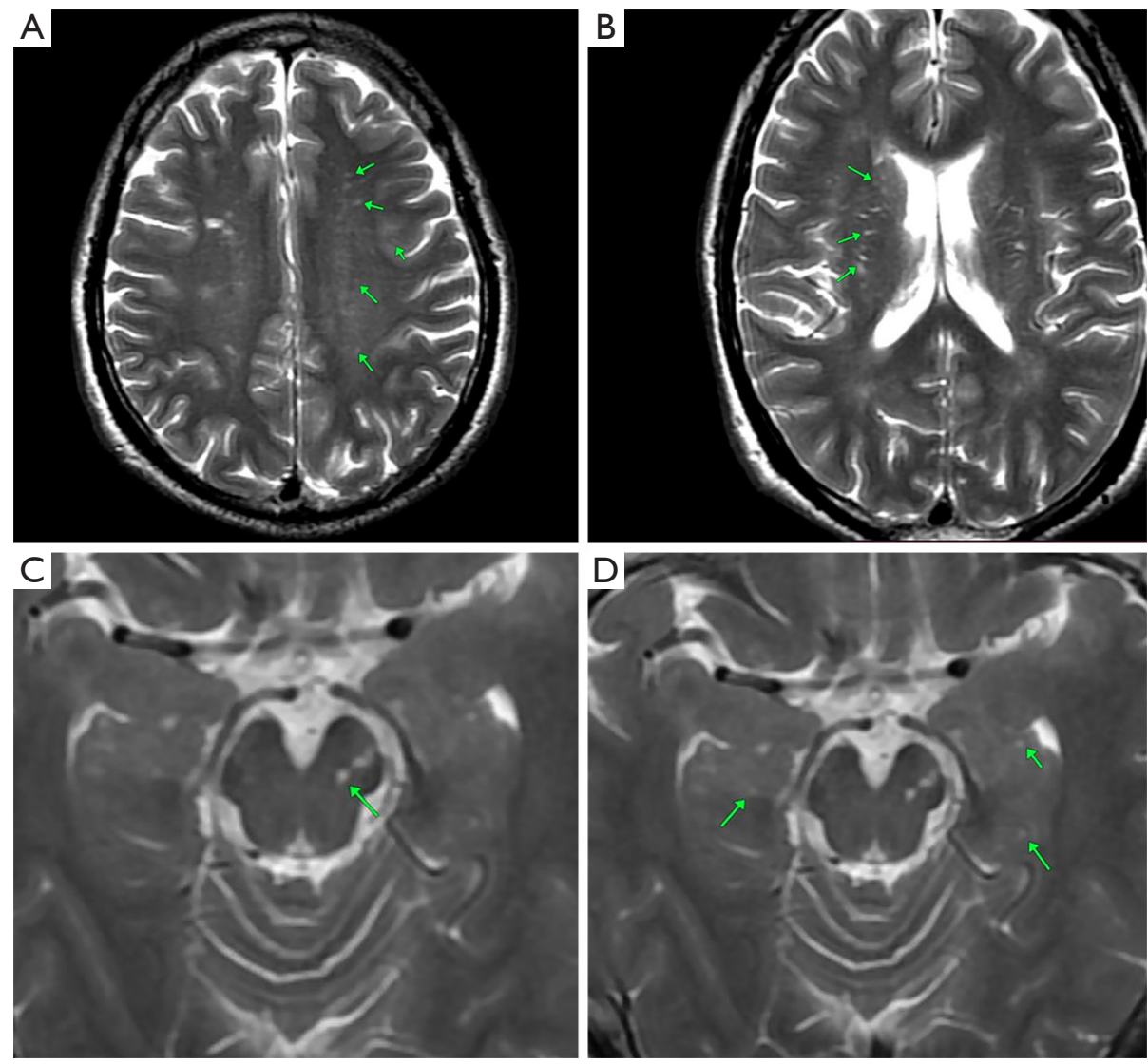

Figure 2 PVSs in different brain regions. (A) CSO-PVS; (B) BG-PVS; (C) midbrain PVS; (D) hippocampus PVS. The green arrows indicate PVSs. CSO, centrum semiovale; PVS, perivascular space; BG, basal ganglia.

The following two-dimensional quantitative indexes of brain atrophy were measured and recorded:

(I) Corpus callosum area (CCA) and corpus callosum index (CCI): measured on the median sagittal image. The area of the corpus callosum was obtained by outlining it. The CCI was measured by hand. The calculation method is to divide the sum of the anterior-to-posterior length of the genu (aa'), the length of the splenium (bb'), and the height of the body of corpus callosum (cc') by the length of the corpus callosum (ab), and the formula is: $\left(\mathrm{aa}^{\prime}+\right.$ $\left.b^{\prime}+c c^{\prime}\right) / a b$.

(II) The ratio of the corpus callosum to cranium (CCR): first, the sagittal area was measured. On the median sagittal image, the dura mater was drawn to outline its intracranial contour, and the dura was shown as a bright line close to the skull intima on the MRI image. In the region in which the dura cannot be seen, the inner edge of the skull was outlined along the substantial part of the brain. Because the inside of the skull at the bottom of the anterior fossa is not visible, the edge of the brain was directly sketched. This line passed directly through the pituitary fossa. The inferior margin of the cranial cavity is defined as the line connecting the anterior and posterior margins of the foramen magnum. CCR $=$ corpus callosum area/median sagittal area.

(III) Width of the third ventricle: the lower border of the corpus callosum was used as the anatomical marker to reconstruct the image, and the width of the third ventricle was measured before and after reconstruction; that is, the unaligned third ventricle width $(\mathrm{u} 3 \mathrm{VW})$ and the aligned third ventricle width (a3VW). The width of the third ventricle was measured at the midpoint of its long axis.

Among these indexes, the numerical values of CCI, CCA, and CCR were negatively correlated with the degree of brain atrophy, and the numerical values of u $3 \mathrm{VW}$ and 

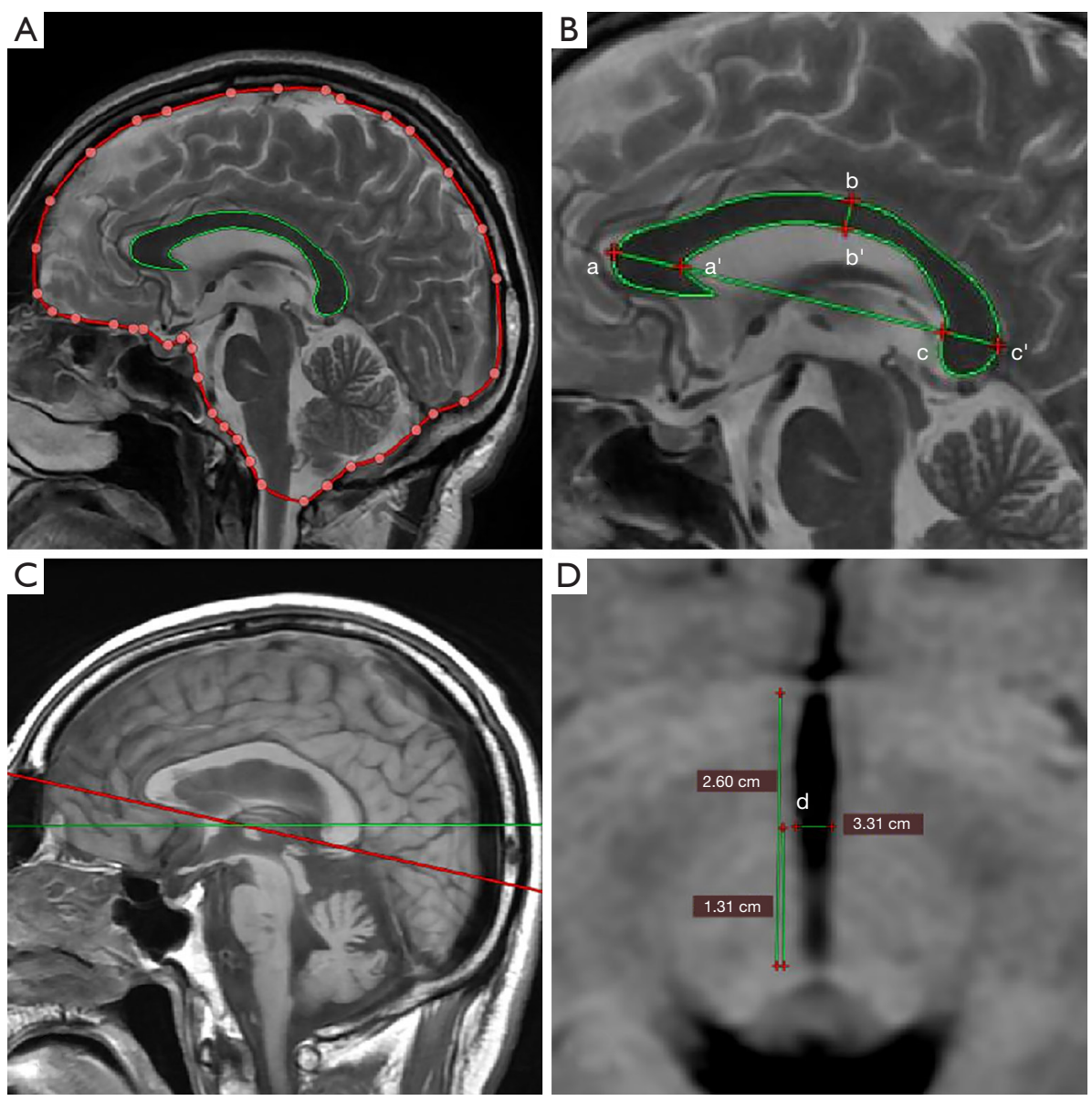

Figure 3 Measurement of brain atrophy. (A) Measurement of CCA (green line) and median sagittal area (red dot line); (B) CCI = (aa' + bb' $\left.+\mathrm{cc}^{\prime}\right) / \mathrm{ab}$; (C) U3VW (green line) and a3VW (red line) are measured on the horizontal plane; (D) the third ventricle width (d) is measured at the midpoint of its length. CCA, corpus callosum area; CCI, corpus callosum index; U3VW, unaligned third ventricle width.

a3VW were positively correlated with the degree of brain atrophy (example: measurement of brain atrophy, see Figure 3).

\section{Statistical analysis}

Statistical analysis was conducted using the statistical software SPSS 21.0. For descriptive statistical results, the Kolmogorov-Smirnov test and visual histogram inspection were used to evaluate the normality of continuous variables. Normally distributed continuous variables were expressed as mean \pm standard deviation $\left(\bar{x}_{ \pm} \mathrm{SD}\right)$, and non-normally distributed continuous variables were expressed as the median and interquartile range (M, IQR). Categorical variables were expressed as percentages (\%) and compared using the chi-squared and Fisher's exact tests. Continuous variables were evaluated using an independent sample $t$-test or Mann-Whitney U test. Univariate and multivariate logistic regression were used to analyze the correlation between PVS and the disability degree of MS. Pearson/ Spearman correlation analysis and partial correlation analysis were used to analyze the correlations of PVS and brain atrophy. The inter-observer agreement was calculated through the Cohen $\kappa$ value and intraclass correlation coefficient (ICC). $\mathrm{P}<0.05$ was considered statistically significant.

\section{Results}

\section{Comparison of general clinical data}

A total of 82 MS patients and 32 healthy controls were 
Table 1 Comparison of general clinical data between the MS group and healthy control group

\begin{tabular}{lccc}
\hline Clinical data & MS group $(\mathrm{n}=82)$ & Control group $(\mathrm{n}=32)$ & $\mathrm{P}$ value \\
\hline Age, years & $37.8 \pm 14.1$ & $38.3 \pm 12.8$ & 0.851 \\
Gender, male & $35(42.7 \%)$ & $9(28.4 \%)$ & 0.151 \\
Hypertension & $18(22 \%)$ & $5(15.6 \%)$ & 0.449 \\
Diabetes mellitus & $6(7.3 \%)$ & $2(3.6 \%)$ & 1.000 \\
Hyperlipidemia & $4(4.7 \%)$ & $0(0 \%)$ & 0.575 \\
Smoking & $15(18.3 \%)$ & $1(3.1 \%)$ & 0.073 \\
Drinking & $4(4.9 \%)$ & $0(0 \%)$ & 0.575 \\
Admission due to EDSS & $2.5[2-4]$ & - & - \\
Duration of disease (months) & $13.5(1-51.75)$ & - & - \\
\hline
\end{tabular}

MS, multiple sclerosis; EDSS, extended disability status scale.

Table 2 Comparison of general clinical data between the acute attack MS group and the remission MS group

\begin{tabular}{lccc}
\hline Clinical data & The acute attack MS group $(\mathrm{n}=59)$ & The remission MS group $(\mathrm{n}=23)$ & $\mathrm{P}$ value \\
\hline Age, years & $39 \pm 14.3$ & $34.7 \pm 13.3$ & 0.221 \\
Gender, male & $23(39.0 \%)$ & $12(52.2 \%)$ & 0.278 \\
Hypertension & $13(22.0 \%)$ & $5(21.7 \%)$ & 0.977 \\
Diabetes mellitus & $4(6.8 \%)$ & $2(8.7 \%)$ & 1.000 \\
Hyperlipidemia & $3(5.1 \%)$ & $1(4.3 \%)$ & 1.000 \\
Smoking & $8(13.6 \%)$ & $7(30.4 \%)$ & 0.066 \\
Drinking & $3(5.1 \%)$ & $1(4.3 \%)$ & 1.000 \\
Duration of disease (months) & $7(0.7-78.0)$ & $30(4.5-70)$ & 0.108 \\
\hline
\end{tabular}

MS, multiple sclerosis.

enrolled in this study. In the MS group, the average age was $37.8 \pm 14.1$ years old, among whom 35 patients were males. In the healthy control group, the average age was $38.3 \pm 12.8$ years old, among whom nine patients were males. The differences in age, gender, hypertension, diabetes, hyperlipidemia, smoking, and drinking between the two groups were not statistically significant $(\mathrm{P}>0.05$ for all, Table 1).

MS patients contained 59 acute attack MS patients and 23 MS patients in remission. The differences in all clinical data between the two groups were not statistically significant $(\mathrm{P}>0.05$ for all, Table 2$)$.

\section{Characteristic analysis of PVS}

The PVS score (4 vs. 3, $\mathrm{P}=0.041$ ), the total number of PVS
$(103.280 \pm 45.107$ vs. $87.625 \pm 30.139, \mathrm{P}=0.035)$, and the total number of EPVS ( 9 vs. $1, \mathrm{P}<0.001$ ) of MS patients were significantly higher than the healthy controls. For the distribution of PVS, the number of PVSs in the BG of MS patients (23.5 vs. $13, \mathrm{P}<0.001)$ was significantly higher than the healthy controls, and the number of PVSs in the CSO of MS patients was higher than the healthy controls (46.5 vs. 43, $\mathrm{P}=0.18$ ), but the difference was not statistically significant. For the distribution of EPVS, the CSO-EPVS ( 3 vs. $0, \mathrm{P}<0.001)$ and BG-EPVS ( 1 vs. 0 , $\mathrm{P}<0.001)$ in the MS group were higher than the healthy control group (Table 3).

The difference of the PVS load was not statistically significant between the acute attack MS patients and MS patients in remission (Table 4).

There were excellent inter-reader agreements for 
Table 3 Comparison of PVS between the MS group and healthy control group

\begin{tabular}{|c|c|c|c|}
\hline PVS & MS group $(n=82)$ & Control group $(n=32)$ & $P$ value \\
\hline \multicolumn{4}{|l|}{ PVS load } \\
\hline PVS score & $4[3-4]$ & $3[3-4]$ & 0.041 \\
\hline Total number of PVSs & $103.280 \pm 45.107$ & $87.625 \pm 30.139$ & 0.035 \\
\hline Total number of EPVS & $9(4.75-17)$ & $1(0.25-2.75)$ & $<0.001$ \\
\hline CSO-PVS & $46.5(27.75-63.5)$ & $43(34.25-60.5)$ & 0.18 \\
\hline BG-PVS & 23.5 [15-36] & $13[7.25-16]$ & $<0.001$ \\
\hline Midbrain PVS & $0(0-1)$ & $0(0-0)$ & 0.64 \\
\hline Hippocampal PVS & $1.5(0-6)$ & $2.5(0-4.75)$ & 0.892 \\
\hline BG-EPVS & $1(0-3.25)$ & $0(0-0)$ & $<0.001$ \\
\hline Midbrain EPVS & $0(0-0)$ & $0(0-0)$ & 0.068 \\
\hline Hippocampal EPVS & $0(0-1)$ & $0(0-0)$ & 0.068 \\
\hline
\end{tabular}

MS, multiple sclerosis; PVS, perivascular space; EPVS, enlarged perivascular space; CSO, centrum semiovale; BG, basal ganglia.

Table 4 Comparison of PVS between the acute attack MS group and the remission MS group

\begin{tabular}{lccc}
\hline PVS load & The acute attack MS group $(n=59)$ & The remission MS group $(n=23)$ & $P$ value \\
\hline PVS score & $4[3-5]$ & $4[3-4]$ & 0.574 \\
Total number of PVSs & $106.3 \pm 51.5$ & $102.1 \pm 42.8$ & 0.703 \\
Total number of EPVS & $7[4-13]$ & $11[5-18]$ & 0.135 \\
\hline
\end{tabular}

MS, multiple sclerosis; PVS, perivascular space; EPVS, enlarged perivascular space.

the identification of PVS (ICC $=0.97, \mathrm{P}<0.001$ ), EPVS (ICC $=0.995, \mathrm{P}<0.001$ ), and PVS score (kappa $=1, \mathrm{P}<0.001$ ).

\section{Comparison of brain atrophy indexes}

The CCI $(0.447 \pm 0.105$ vs. $0.502 \pm 0.060, \mathrm{P}=0.001)$, CCA $\left(5.382 \pm 1.380\right.$ vs. $\left.6.147 \pm 1.114 \mathrm{~cm}^{2}, \mathrm{P}=0.006\right), \mathrm{CCR}$ ( $0.037 \pm 0.009$ vs. $0.043 \pm 0.007, \mathrm{P}=0.006)$ of MS patients were lower than those of healthy controls, but the u $3 \mathrm{VW}$ $(4.404 \pm 2.711$ vs. $3.024 \pm 0.928 \mathrm{~mm}, \mathrm{P}=0.006)$ and $\mathrm{a} 3 \mathrm{VW}$ (4.428 \pm 2.687 vs. $3.035 \pm 0.953 \mathrm{~mm}, \mathrm{P}=0.005$ ) of $\mathrm{MS}$ patients were significantly higher than those of the healthy control group (Table 5).

The difference of all brain atrophy indexes was not statistically significant between the acute attack MS patients and MS patients in remission (Table 6).

\section{Correlation analysis of PVS with brain atrophy and MS disability}

Univariate binary logistic regression analysis revealed that the severity of disability was not correlated with the number, size, distribution of PVS in acute MS patients. After controlling for gender, age, hypertension, recurrence frequency, and duration of disease, the severity of disability remained uncorrelated with the number, size, distribution of P VS.

Univariate logistic regression analysis revealed that CCI (OR: 0.495; 95\% CI: 0.296-0.828; P=0.007), CCA (OR: 0.677; 95\% CI: 0.479-0.957; P=0.027), CCR (OR: 0.515, 95\% CI: 0.303-0.849, $\mathrm{P}=0.009$ ), u3VW (OR: 1.437; 95\% CI: $1.051-1.963 ; \mathrm{P}=0.023$ ), and a3VW (OR: $1.487 ; 95 \%$ CI: $1.084-2.038 ; \mathrm{P}=0.014)$ were associated with the severity of disability, and, after controlling for gender and age, CCI 
Table 5 Comparison of brain atrophy indexes between the MS group and healthy control group

\begin{tabular}{lccc}
\hline Brain atrophy indexes & MS group $(\mathrm{n}=82)$ & Control group $(\mathrm{n}=32)$ & $\mathrm{P}$ value \\
\hline $\mathrm{CCl}$ & $0.447 \pm 0.105$ & $0.502 \pm 0.060$ & 0.001 \\
$\mathrm{CCA}, \mathrm{cm}^{2}$ & $5.382 \pm 1.380$ & $6.147 \pm 1.114$ & 0.006 \\
$\mathrm{CCR}$ & $0.037 \pm 0.009$ & $0.043 \pm 0.007$ & 0.001 \\
u3VW, mm & $4.404 \pm 2.711$ & $3.024 \pm 0.928$ & 0.006 \\
a3VW, $\mathrm{mm}$ & $4.428 \pm 2.687$ & $3.035 \pm 0.953$ & 0.005 \\
\hline
\end{tabular}

MS, multiple sclerosis; CCI, corpus callosum index; CCA, corpus callosum area; CCR, ratio of the corpus callosum to the cranium; a3VW, aligned third ventricle width; u3VW, unaligned third ventricle width.

Table 6 Comparison of brain atrophy indexes between the acute attack MS group and the remission MS group

\begin{tabular}{lccc}
\hline Brain atrophy indexes & The acute attack MS group $(n=59)$ & The remission MS group $(n=23)$ & $P$ value \\
\hline $\mathrm{CCl}$ & $0.445 \pm 0.103$ & $0.451 \pm 0.113$ & 0.832 \\
$\mathrm{CCA}, \mathrm{cm}^{2}$ & $5.446 \pm 1.358$ & $5.219 \pm 1.451$ & 0.507 \\
$\mathrm{CCR}$ & $0.038 \pm 0.009$ & $0.036 \pm 0.009$ & 0.422 \\
u3VW, mm & $4.063 \pm 1.525$ & $5.279 \pm 4.452$ & 0.212 \\
a3VW, $\mathrm{mm}$ & $4.093 \pm 1.538$ & $5.286 \pm 4.390$ & 0.215 \\
\hline
\end{tabular}

MS, multiple sclerosis; $\mathrm{CCl}$, corpus callosum index; CCA, corpus callosum area; CCR, ratio of the corpus callosum to the cranium; a3VW, aligned third ventricle width; u3VW, unaligned third ventricle width.

(OR: 0.479; 95\% CI: 0.284-0.808; $\mathrm{P}=0.006)$, CCA (OR: 0.619; 95\% CI: 0.423-0.906; P=0.013), CCR (OR: 0.466; 95\% CI: 0.274-0.792; P=0.005), u3VW (OR: 1.446; 95\% CI: $1.055-1.981 ; \mathrm{P}=0.022)$, and a3VW (OR: $1.499 ; 95 \% \mathrm{CI}$ : $1.090-2.061 ; \mathrm{P}=0.013)$ were still associated with the severity of disability (Table 7).

\section{Correlation between PVS and brain atrophy}

In MS patients, the PVS score ( $\mathrm{rs}=0.352 ; \mathrm{P}=0.01)$, the total number of PVSs ( $\mathrm{rs}=0.353 ; \mathrm{P}=0.001)$, and the total number of EPVSs ( $\mathrm{rs}=0.258 ; \mathrm{P}=0.019$ ) were correlated with age, and the PVS score ( $\mathrm{rs}=0.296 ; \mathrm{P}=0.007)$ and total number of PVSs ( $r s=0.289 ; \mathrm{P}=0.008$ ) were associated with hypertension, but not with recurrence times, duration of disease, EDSS scores, and other clinical data. In MS patients, the PVS score was correlated with CCA ( $\mathrm{rs}=0.272 ; \mathrm{P}=0.013)$ and $\mathrm{CCR}(\mathrm{rs}=0.219$; $\mathrm{P}=0.048)$. The total number of PVSs was associated with CCA ( $\mathrm{rs}=0.255 ; \mathrm{P}=0.021$ ), and had a positive correlation tendency with CCR ( $\mathrm{rs}=0.201 ; \mathrm{P}=0.071)$, $\mathrm{u} 3 \mathrm{VW}(\mathrm{rs}=-0.199$; $\mathrm{P}=0.073)$, and a3VW ( $\mathrm{rs}=-0.201 ; \mathrm{P}=0.071)$. In the healthy control group, the PVS score was only correlated with males $(\mathrm{rs}=0.437 ; \mathrm{P}=0.012)$ and not correlated with other clinical data and brain atrophy. The total number of PVSs and EPVSs were not associated with the general clinical data and brain atrophy. After adjustment of hypertension and age, this correlation disappeared (Table 8).

In patients with acute attack MS, the total number of PVS (age: $r s=0.401, \mathrm{P}=0.002$; hypertension: $\mathrm{rs}=0.303$, $\mathrm{P}=0.02$ ), the total number of EPVS (age: $\mathrm{rs}=0.359, \mathrm{P}=0.005$; hypertension: $r s=0.261, \mathrm{P}=0.046$ ), $\mathrm{PVS}$ score (age: $\mathrm{rs}=0.349$, $\mathrm{P}=0.007$; hypertension: $\mathrm{rs}=0.300, \mathrm{P}=0.021$ ) were related to age and hypertension rather to other clinical factors and brain atrophy indexes. In MS patients in remission, the total number of PVSs was correlated with CCA ( $\mathrm{rs}=0.487$; $\mathrm{P}=0.019)$, CCR ( $\mathrm{rs}=0.479 ; \mathrm{P}=0.021$ ), and PVS score was correlated with $\mathrm{CCA}(\mathrm{rs}=0.453 ; \mathrm{P}=0.030)$. After adjustment of hypertension and age, the total number of PVSs was correlated with CCA ( $\mathrm{rs}=0.419 ; \mathrm{P}=0.049$ ) (Table 9).

\section{Discussion}

The PVS load of MS patients is heavier, which is usually considered an imaging marker of MS inflammatory response

The present study revealed that, compared with healthy 
Table 7 Correlation analysis of PVS with brain atrophy linear indexes and the severity of disability in MS

\begin{tabular}{|c|c|c|c|c|c|c|}
\hline Variables & \multicolumn{3}{|c|}{ Univariate analysis } & \multicolumn{3}{|c|}{ Multivariate analysis } \\
\hline PVS score & 0.452 & 0.843 & $0.54-1.316$ & $0.344^{*}$ & 0.775 & $0.458-1.313$ \\
\hline Total number of PVSs & 0.898 & 1.001 & $0.989-1.013$ & $0.874^{\star}$ & 1.001 & $0.987-1.016$ \\
\hline Total number of EPVS & 0.283 & 0.97 & $0.917-1.026$ & $0.41^{*}$ & 0.973 & $0.913-1.038$ \\
\hline $\mathrm{CCA}, \mathrm{cm}^{2}$ & 0.027 & 0.677 & $0.479-0.957$ & $0.013^{\#}$ & 0.619 & $0.423-0.906$ \\
\hline CCR & 0.009 & 0.515 & $0.303-0.849$ & $0.005^{\#}$ & 0.466 & $0.274-0.792$ \\
\hline u3vW, mm & 0.023 & 1.437 & $1.051-1.963$ & $0.022^{\#}$ & 1.446 & $1.055-1.981$ \\
\hline a3VW, mm & 0.014 & 1.487 & $1.084-2.038$ & $0.013^{\#}$ & 1.499 & $1.090-2.061$ \\
\hline
\end{tabular}

*, after controlling for gender, age, hypertension, recurrence frequency and duration of disease; ", after controlling for gender and age. MS, multiple sclerosis; PVS, perivascular space; EPVS, enlarged perivascular space; CCl, corpus callosum index; CCA, corpus callosum area; $\mathrm{CCR}$, ratio of the corpus callosum to the cranium; a3VW, aligned third ventricle width; u3VW, unaligned third ventricle width.

controls, the PVS load of MS patients was heavier. That is, the PVS score was higher, the number and diameter were larger, and the PVS load of BG and CSO was especially heavy.

At present, inconsistent results of the frequency, number, volume, and significance of PVS in MS have been published. First, Achiron and Faibel found a high PVS load in a group of newly diagnosed MS patients, and it is considered that it may be a neuroradiological marker reflecting the early inflammatory changes of MS (11). Most neuroimaging studies on PVS in MS patients have consistently revealed that the number $(12,13,17)$ and size (14) of PVS in MS patients were higher than those in healthy controls. However, an Arab study failed to replicate these findings (15). The most consistent site of PVS with MS was the CSO $(11,13,16,17)$. Our previous study found that the incidence of PVS in the BG was the highest in MS patients. Compared with the control group, EPVS seemed to be more common at atypical sites in MS patients (19). The results of the present study revealed that PVS scores and the number and size of PVS in MS patients were higher than those in healthy controls. That is, the PVS load was higher in MS patients than in healthy controls. The number of PVSs in the BG of MS patients was significantly larger than in healthy controls. The number of PVSs in the CSO was larger than in healthy controls. The number of PVSs in the CSO and BG was larger than in healthy controls. PVSs are a pathway for cerebral lymphatic drainage between the periarterial space and the lymphatic system, and are implicated in the case of blood-brain barrier abnormality and inflammation. During the onset of MS, the immuneinflammatory cells are widely distributed in the PVS, and inflammatory activity leads to cell infiltration and edema, which may lead to PVS enlargement, thus increasing the number and volume of PVS on MRI. Macrophages carrying MHC-II antigens can capture foreign antigens entering the brain in PVS, which are treated locally by lymphocytes or transported to local lymph nodes $(20,21)$. The macrophages contained in PVS have contact with the circulating $\mathrm{T}$ lymphocytes (22) which the immune response starts from. $\mathrm{T}$ cell activation begins at the periphery of the lymphoid compartment and then reaches the CNS circulating in the PVS (14). Once the large number of activated macrophages and T and B lymphocytes accumulate in PVS, a comprehensive immune response can be generated there. This perivascular accumulation of cells could be due to the resultant overflow of immune response, or the stimulating antigens being confined to cells in the PVSs. In addition, the endocytosis of perivascular macrophages can cause an immune response in PVS and identify endogenous serum proteins (IgG, complement C9, IgM) in P VS. The high PVS load of MS revealed in this study may be related to the inflammatory reaction of P VS. Furthermore, the high PVS load in the CSO and BG may indicate that perivascular macrophages and stimulating antigens at this site have more significant effects and more active immune-mediated responses. Studies have shown that the PVS load of MS patients in the acute attack stage $(11,14)$ and remission stage (12) were higher than that of the healthy control group. However, there was still a lack of reports on the 
Table 8 Correlation analysis of PVS and brain atrophy linear indexes in the MS group and healthy control group

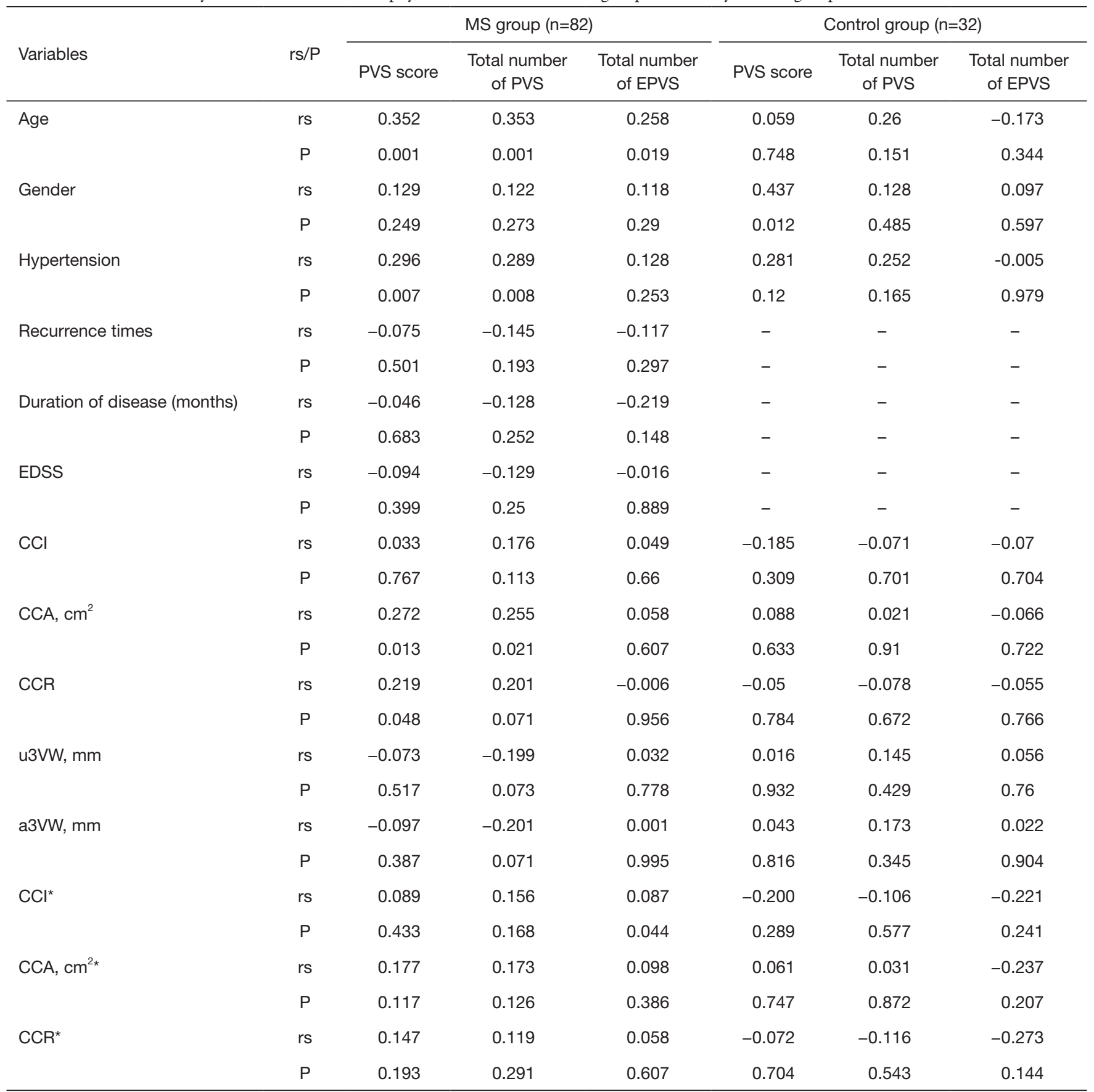

*, partial correlation analysis after adjusting for age and hypertension. MS, multiple sclerosis; PVS, perivascular space; EPVS, enlarged perivascular space; EDSS, extended disability status scale; $\mathrm{CCI}$, corpus callosum index; CCA, corpus callosum area; CCR, ratio of the corpus callosum to the cranium; a3VW, aligned third ventricle width; u3VW, unaligned third ventricle width. 
Table 9 Correlation analysis of PVS and brain atrophy linear indexes in the acute attack MS group and the remission MS group

\begin{tabular}{|c|c|c|c|c|c|c|c|}
\hline Variables & $\mathrm{rs} / \mathrm{P}$ & \multicolumn{3}{|c|}{ The acute attack MS group $(n=59)$} & \multicolumn{3}{|c|}{ The remission MS group $(n=23)$} \\
\hline Age & $\mathrm{P}$ & 0.007 & 0.002 & 0.005 & 0.068 & 0.198 & 0.557 \\
\hline \multirow[t]{2}{*}{ Hypertension } & rs & 0.300 & 0.303 & 0.261 & 0.262 & 0.238 & -0.175 \\
\hline & $P$ & 0.021 & 0.02 & 0.046 & 0.226 & 0.273 & 0.424 \\
\hline Recurrence times & rs & -0.082 & -0.081 & -0.151 & 0.015 & -0.140 & 0.109 \\
\hline \multirow[t]{2}{*}{ EDSS } & rs & -0.002 & -0.131 & -0.189 & -0.266 & -0.173 & -0.26 \\
\hline & $\mathrm{P}$ & 0.990 & 0.321 & 0.153 & 0.220 & 0.431 & 0.231 \\
\hline \multirow[t]{2}{*}{$\mathrm{CCl}$} & rs & -0.017 & 0.077 & 0.102 & 0.146 & 0.372 & -0.098 \\
\hline & $\mathrm{P}$ & 0.896 & 0.560 & 0.444 & 0.505 & 0.080 & 0.656 \\
\hline \multirow[t]{2}{*}{$\mathrm{CCA}, \mathrm{cm}^{2}$} & rs & 0.213 & 0.149 & 0.032 & 0.453 & 0.487 & 0.058 \\
\hline & $\mathrm{P}$ & 0.105 & 0.260 & 0.808 & 0.030 & 0.019 & 0.791 \\
\hline Ratio of corpus callosum to cranium & rs & 0.178 & 0.083 & -0.03 & 0.325 & 0.479 & -0.046 \\
\hline \multirow[t]{2}{*}{$\mathrm{CCl}^{*}$} & rs & 0.077 & 0.079 & 0.122 & 0.127 & 0.314 & 0.117 \\
\hline & $P$ & 0.567 & 0.557 & 0.365 & 0.584 & 0.166 & 0.614 \\
\hline \multirow[t]{2}{*}{$\mathrm{CCA}, \mathrm{cm}^{2 *}$} & rs & 0.144 & 0.075 & 0.031 & 0.313 & 0.419 & 0.301 \\
\hline & $\mathrm{P}$ & 0.286 & 0.581 & 0.818 & 0.166 & 0.049 & 0.185 \\
\hline \multirow[t]{2}{*}{ Ratio of corpus callosum to cranium* } & rs & 0.118 & 0.005 & -0.012 & 0.279 & 0.414 & 0.259 \\
\hline & $\mathrm{P}$ & 0.381 & 0.971 & 0.928 & 0.220 & 0.062 & 0.257 \\
\hline
\end{tabular}

*, partial correlation analysis after adjusting for age and hypertension. MS, multiple sclerosis; PVS, perivascular space; EPVS, enlarged perivascular space; EDSS, extended disability status scale; CCI, corpus callosum index; CCA, corpus callosum area; CCR, ratio of the corpus callosum to the cranium; a3VW, aligned third ventricle width; u3VW, unaligned third ventricle width. 
comparison of PVS between the two groups. This study found that the number and size of PVS in patients with MS in remission did not differ from those in the acute phase. This might be because the expansion of the PVS in the acute attack phase was not compensated in the remission phase, so there is still the possibility of identifying more PVS, therefore it didn't reflect the continuation of inflammatory activity.

This study revealed that, in MS patients, the PVS score and total PVS number were correlated with age and hypertension; the number of EPVS was related to age and not gender. There was no correlation between the PVS score, the total number of PVS, and the number of EPVS with the EDSS score, recurrence times, and disease duration. In acute attack MS patients, the total PVS and EPVS number, PVS score were related to age and hypertension. In MS patients in remission, the total PVS number was correlated with CCA, CCR, and PVS score was correlated with CCA. Previous studies revealed that the correlation between PVS and MS clinical features showed that the results were not consistent. Although a minority of prior studies indicated that PVS was significantly correlated with age (17), course of disease $(13,17)$, and gender (16), other studies have not found this correlation $(11,14)$. Most of the studies did not report that PVS was associated with MS physical disability $(11,14,17)$ or transition to a progressive type (13). Current studies have not demonstrated that PVS could be used as an imaging marker for the severity of MS. This is consistent with the results of this study.

\section{The severity of brain atrophy in MS patients was more severe, and there was a correlation between PVS and MS brain atrophy}

Brain atrophy in MS occurs in the early stage of the disease, which can be found in all clinical stages of MS and develops gradually with the appearance of inflammatory lesions. Brain atrophy in MS represents a destructive and irreversible pathological process $(23,24)$. Brain atrophy is widely considered an effective, sensitive, and repeatable indicator of neurodegenerative changes in MS (3).

The present study revealed that the severity of brain atrophy in MS patients was higher than in healthy controls. CCI, CCA, CCR, u3VW, and a $3 \mathrm{VW}$ were associated with the severity of disability in MS patients. Brain atrophy in MS may reflect inflammation-induced axonal loss and subsequent Wallerian degeneration and post-inflammation neurodegeneration. This may be partly due to the failure of myelin regeneration. The mechanism of brain atrophy in MS remains unclear. It is generally considered to be the result of extensive axonal transection and demyelination (25). CCI, CCA, and CCR are effective measurement indicators of corpus callosum atrophy, and corpus callosum atrophy is an effective biomarker of MS (26). A previous study has revealed that CCA and CCI were related to EDSS (27). This is consistent with the results of this study. The volume of brain parenchyma decreased; that is, the atrophy of the cortex and medulla, which was accompanied by the corresponding increase of the space volume of the CSF. The third ventricle width $(3 \mathrm{VW})$ has been considered a reliable marker for quantifying central brain atrophy using MRI or transcranial ultrasound (28). Some studies have revealed that the accuracy of the $3 \mathrm{VW}$ in MS measured by the plane survey was similar to that of the semi-automatic volume measurement of the third ventricle. The enlargement of the third ventricle was associated with atrophy of adjacent structures (especially the thalamus), which may be an indirect indicator of thalamic atrophy in MS (29). There are different results on the correlation between $3 \mathrm{VW}$ and EDSS. Previous studies revealed that central brain atrophy was not related to the EDSS score (30,31). Another study observed that central brain atrophy in MS patients was correlated with the EDSS score (32). The results of this study support that $3 \mathrm{VW}$ is associated with EDSS in MS patients.

The present study revealed that in MS patients, PVS was correlated with age and hypertension. Previous studies indicated that PVS was significantly associated with age, suggesting that PVS may be a manifestation of brain aging. With the decline of brain clearance function and the atrophy of brain parenchyma, the PVS load increases. Hypertension is a risk factor for PVS, and the mechanism may be that when blood pressure suddenly rises beyond the limit of cerebral vascular self-regulation, the cerebrovascular fluid leaks into PVS through the blood-brain barrier; thus, the number and volume of PVSs that can be detected increase $(33,34)$.

This study revealed that the PVS scores of MS patients were correlated with CCA and CCR, and the total number of PVSs was correlated with CCA. However, after adjustment of hypertension and age, this correlation disappeared. Analysis in subgroups shown that the total number of PVSs was correlated with CCA and CCR, and PVS score was correlated with CCA in MS patients in remission, after adjustment of hypertension and age, 
the correlation of the total number of PVSs and CCA remained. It was shown that PVS in MS might be related to brain atrophy to a certain extent, and PVS may be related to the neurodegeneration of MS. In previous studies, Kilsdonk et al. revealed in their 7T MRI study that a higher EPVS count was associated with supratentorial brain atrophy rather than whole-brain atrophy (17). Other studies using low field MRI (i.e., 1.5T or 3T) failed to repeat this finding. The corpus callosum is a transverse nerve fiber bundle that connects the two hemispheres of the brain and provides communication between the two hemispheres, mainly consisting of myelinated nerve fibers. CCA in MS is considered to be the result of focal demyelination and axonal injury, but it is also considered to be the result of Wallerian degeneration and distal demyelination of deep white matter to axon transection after inflammation (27). A study pointed out that CCA and CCI were more sensitive and specific than MRI measurements of craniocerebral volume, and they were relatively fast and simple, and the CCR may further adjust the differences among individuals (35). A previous study revealed that CCA was associated with whole-brain atrophy and gray matter atrophy in MS and associated with white matter lesions in MS; that is, the lesions of gray matter and white matter in MS all affected corpus callosum (36). CCA is disproportionately low in MS, which shows a larger effect than other atrophy indexes, and the degree of CCA was significantly higher than other common global atrophy indicators [such as brain parenchymal fraction (BPF), gray matter fraction (GMF), and white matter fraction (WMF)], and the corpus callosum may be a sensitive tool for monitoring the destructive aspects of the disease. This may be the reason why this study could repeat this result with low field intensity and a two-dimensional brain atrophy measurement index.

The damage of lymphoid pathways is a risk factor for the development of neurodegenerative diseases. In neurodegenerative diseases, the decrease of the waste clearance function of PVS and the accumulation of neurotoxic substances promote each other, resulting in the occurrence and progress of the disease (37). Among the various causes of progression in Alzheimer's disease, extracellular $\mathrm{A} \beta$ protein accumulation, neuroinflammation, oxidative stress, and apoptosis play major roles (38). However, current studies on brain atrophy in MS suggest that the pathogenic factors of MS brain atrophy change with the progress of the disease. In the early stage of the disease, brain atrophy is mainly the result of inflammatory changes, and in the late stages of the disease, the causes of brain atrophy are rarely inflammatory changes and mostly neurodegenerative changes. Inflammation may be an important factor of whole brain tissue loss in the early stage of the disease [clinically isolated syndrome (CIS)]. With the progress of the disease, other mechanisms are partially independent of inflammatory demyelinating lesions in white matter, such as activation of microglia in normal-appearing white matter (NAWM), meningitis, iron deposition, oxidative stress, and diffuse axonal injury (39). A biopsy study also confirmed that even in the absence of inflammation, atrophy might continue (40). The level of iron in NAWM of patients with progressive MS was significantly lower (41). Iron is essential for myelin synthesis and neurogenesis, and iron deficiency in normal tissues may further promote diffuse axonal loss and CNS atrophy in MS. Bocheng et al. revealed that in the animal model of ischemia of the ventricle, the ferritin level around the ventricle of mice in the deep cervical lymph node (DCLN) resection group was higher than in the DCLN preservation group (42). Because DCLN is considered to be downstream of the lymphatic system, this result indicates that the function of the lymphoid system may be related to iron excretion, and PVS is an important part of the lymphoid system. Zhou et al. revealed that the local iron deposition in normal brain tissue in the elderly was related to the function of the lymphoid system, and iron deposition may further lead to dysfunction of the lymphoid system (43). In summary, it is assumed that, in the early stages of MS, the inflammatory immune cells enter the PVS, which may lead to the increase of PVS, and with the progress of the disease, the degenerative lesions in MS develop gradually. On the one hand, its degenerative mechanism may lead to the impairment of PVS clearance function, leading to the expansion of PVS, while on the other hand, atrophy of brain parenchyma stretches the PVS to expand.

\section{Limitations}

The present study has certain limitations. First, brain atrophy in MS patients was affected by the stage of the disease (although this study shown no difference between brain atrophy indexes of acute attack MS patients and MS patients in remission), further researches are needed to study the association of PVS in MS patients with brain atrophy and disease severity/disease progression. The correlation between PVS and inflammatory and/or degenerative changes in MS still needs to be verified and 
replicated. Second, in this study, a manual method was used to interpret PVS, which may cause bias in interpretation. Finally, this study is a retrospective study, and in the collection process, patients with incomplete clinical data or imaging data may be included.

\section{Conclusions}

In summary, the PVS load in MS is heavier than the healthy control group; that is, the PVS score is higher, the number and diameter are larger, and the increased load of PVS is mainly in the BG and CSO. In MS patients, PVS is correlated with age, hypertension, CCA, and CCR, but not with the EDSS score and disease duration.

\section{Acknowledgments}

We are particularly grateful to all the people who have given us help on our article.

Funding: This study was funded by the National Natural Science Foundation of China (No. 81471228) and Hebei Medical Science Research Project (No. 20200048).

\section{Footnote}

Reporting Checklist: The authors have completed the MDAR checklist. Available at https://dx.doi.org/10.21037/qims21-705

Conflicts of Interest: All authors have completed the ICMJE uniform disclosure form (available at https://dx.doi. org/10.21037/qims-21-705). The authors have no conflicts of interest to declare.

Ethical Statement: The authors are accountable for all aspects of the work in ensuring that questions related to the accuracy or integrity of any part of the work are appropriately investigated and resolved. The study was conducted in accordance with the Declaration of Helsinki (as was revised in 2013). The study was approved by Ethics Committee of the Second Hospital of Hebei Medical University (No. 2021-R433) and informed consent was taken from all the patients.

Open Access Statement: This is an Open Access article distributed in accordance with the Creative Commons Attribution-NonCommercial-NoDerivs 4.0 International License (CC BY-NC-ND 4.0), which permits the non- commercial replication and distribution of the article with the strict proviso that no changes or edits are made and the original work is properly cited (including links to both the formal publication through the relevant DOI and the license). See: https://creativecommons.org/licenses/by-nc-nd/4.0/.

\section{References}

1. de Vries HE, Kuiper J, de Boer AG, Van Berkel TJ, Breimer DD. The blood-brain barrier in neuroinflammatory diseases. Pharmacol Rev 1997;49:143-55.

2. Troili F, Cipollini V, Moci M, Morena E, Palotai M, Rinaldi V, Romano C, Ristori G, Giubilei F, Salvetti M, Orzi F, Guttmann CRG, Cavallari M. Perivascular Unit: This Must Be the Place. The Anatomical Crossroad Between the Immune, Vascular and Nervous System. Front Neuroanat 2020;14:17.

3. Maclullich AM, Wardlaw JM, Ferguson KJ, Starr JM, Seckl JR, Deary IJ. Enlarged perivascular spaces are associated with cognitive function in healthy elderly men. J Neurol Neurosurg Psychiatry 2004;75:1519-23.

4. Hiroki M, Miyashita K. Linear hyperintensity objects on magnetic resonance imaging related to hypertension. Cerebrovasc Dis 2001;11:164-8.

5. Banerjee G, Kim HJ, Fox Z, Jäger HR, Wilson D, Charidimou A, Na HK, Na DL, Seo SW, Werring DJ. MRI-visible perivascular space location is associated with Alzheimer's disease independently of amyloid burden. Brain 2017;140:1107-16.

6. Ramirez J, Berezuk C, McNeely AA, Gao F, McLaurin J, Black SE. Imaging the Perivascular Space as a Potential Biomarker of Neurovascular and Neurodegenerative Diseases. Cell Mol Neurobiol 2016;36:289-99.

7. Healy BC, Buckle GJ, Ali EN, Egorova S, Khalid F, Tauhid S, Glanz BI, Chitnis T, Guttmann CRG, Weiner HL, Bakshi R. Characterizing Clinical and MRI Dissociation in Patients with Multiple Sclerosis. J Neuroimaging 2017;27:481-5.

8. Davis FA. The clinico-radiological paradox in multiple sclerosis: novel implications of lesion size. Mult Scler 2014;20:515-6.

9. Trapp BD, Nave KA. Multiple sclerosis: an immune or neurodegenerative disorder? Annu Rev Neurosci 2008;31:247-69.

10. Calabrese M, Atzori M, Bernardi V, Morra A, Romualdi C, Rinaldi L, McAuliffe MJ, Barachino L, Perini P, Fischl B, Battistin L, Gallo P. Cortical atrophy is relevant in multiple 
sclerosis at clinical onset. J Neurol 2007;254:1212-20.

11. Achiron A, Faibel M. Sandlike appearance of VirchowRobin spaces in early multiple sclerosis: a novel neuroradiologic marker. AJNR Am J Neuroradiol 2002;23:376-80.

12. Conforti R, Cirillo M, Saturnino PP, Gallo A, Sacco R, Negro A, Paccone A, Caiazzo G, Bisecco A, Bonavita S, Cirillo S. Dilated Virchow-Robin spaces and multiple sclerosis: 3 T magnetic resonance study. Radiol Med 2014;119:408-14.

13. Cavallari M, Egorova S, Healy BC, Palotai M, Prieto JC, Polgar-Turcsanyi M, Tauhid S, Anderson M, Glanz B, Chitnis T, Guttmann CRG. Evaluating the Association between Enlarged Perivascular Spaces and Disease Worsening in Multiple Sclerosis. J Neuroimaging 2018;28:273-7.

14. Wuerfel J, Haertle M, Waiczies H, Tysiak E, Bechmann I, Wernecke KD, Zipp F, Paul F. Perivascular spaces-MRI marker of inflammatory activity in the brain? Brain 2008;131:2332-40.

15. Al-Saeed O, Athyal R, Ismail M, Sheikh M. Significance of Virchow-Robin spaces in patients newly diagnosed with multiple sclerosis: a case-control study in an Arab population. Med Princ Pract 2012;21:447-51.

16. Etemadifar M, Hekmatnia A, Tayari N, Kazemi M, Ghazavi A, Akbari M, Maghzi AH. Features of VirchowRobin spaces in newly diagnosed multiple sclerosis patients. Eur J Radiol 2011;80:e104-8.

17. Kilsdonk ID, Steenwijk MD, Pouwels PJ, Zwanenburg JJ, Visser F, Luijten PR, Geurts J, Barkhof F, Wattjes MP. Perivascular spaces in MS patients at 7 Tesla MRI: a marker of neurodegeneration? Mult Scler 2015;21:155-62.

18. Potter GM, Chappell FM, Morris Z, Wardlaw JM. Cerebral perivascular spaces visible on magnetic resonance imaging: development of a qualitative rating scale and its observer reliability. Cerebrovasc Dis 2015;39:224-31.

19. Kwee RM, Kwee TC. Virchow-Robin spaces at MR imaging. Radiographics 2007;27:1071-86.

20. Brownell B, Hughes JT. The distribution of plaques in the cerebrum in multiple sclerosis. J Neurol Neurosurg Psychiatry 1962;25:315-20.

21. Esiri MM, Gay D. Immunological and neuropathological significance of the Virchow-Robin space. J Neurol Sci 1990;100:3-8.

22. Groeschel S, Chong WK, Surtees R, Hanefeld F. VirchowRobin spaces on magnetic resonance images: normative data, their dilatation, and a review of the literature. Neuroradiology 2006;48:745-54.
23. Ge Y, Grossman RI, Udupa JK, Wei L, Mannon LJ, Polansky M, Kolson DL. Brain atrophy in relapsingremitting multiple sclerosis and secondary progressive multiple sclerosis: longitudinal quantitative analysis. Radiology 2000;214:665-70.

24. Barnett Y, Garber JY, Barnett MH. MRI biomarkers of disease progression in multiple sclerosis: old dog, new tricks? Quant Imaging Med Surg 2020;10:527-32.

25. Amiri H, de Sitter A, Bendfeldt K, Battaglini M, Gandini Wheeler-Kingshott CAM, Calabrese M, Geurts JJG, Rocca MA, Sastre-Garriga J, Enzinger C, de Stefano N, Filippi M, Rovira Á, Barkhof F, Vrenken H; MAGNIMS Study Group. Urgent challenges in quantification and interpretation of brain grey matter atrophy in individual MS patients using MRI. Neuroimage Clin 2018;19:466-75.

26. Vaneckova M, Kalincik T, Krasensky J, Horakova D, Havrdova E, Hrebikova T, Seidl Z. Corpus callosum atrophy--a simple predictor of multiple sclerosis progression: a longitudinal 9-year study. Eur Neurol 2012;68:23-7.

27. Klawiter EC, Ceccarelli A, Arora A, Jackson J, Bakshi S, Kim G, Miller J, Tauhid S, von Gizycki C, Bakshi $\mathrm{R}$, Neema M. Corpus callosum atrophy correlates with gray matter atrophy in patients with multiple sclerosis. J Neuroimaging 2015;25:62-7.

28. Benedict RH, Weinstock-Guttman B, Fishman I, Sharma J, Tjoa CW, Bakshi R. Prediction of neuropsychological impairment in multiple sclerosis: comparison of conventional magnetic resonance imaging measures of atrophy and lesion burden. Arch Neurol 2004;61:226-30.

29. Lutz T, Bellenberg B, Schneider R, Weiler F, Köster O, Lukas C. Central Atrophy Early in Multiple Sclerosis: Third Ventricle Volumetry versus Planimetry. J Neuroimaging 2017;27:348-54.

30. Audoin B, Davies GR, Finisku L, Chard DT, Thompson AJ, Miller DH. Localization of grey matter atrophy in early RRMS : A longitudinal study. J Neurol 2006;253:1495-501.

31. Hughes S, Spelman T, Trojano M, Lugaresi A, Izquierdo G, Grand'maison F,, et al. The Kurtzke EDSS rank stability increases 4 years after the onset of multiple sclerosis: results from the MSBase Registry. J Neurol Neurosurg Psychiatry 2012;83:305-10.

32. Müller M, Esser R, Kötter K, Voss J, Müller A, Stellmes P. Third ventricular enlargement in early stages of multiple sclerosis is a predictor of motor and neuropsychological deficits: a cross-sectional study. BMJ Open 2013;3:e003582. 
33. Zhang HJ. Analysis of dilated perivascular space and dynamic blood pressure variability in patients with first lacunar infarction. Chinese Journal of Practical Nervous Diseases 2017;20:89-91.

34. Dai SP, Pang J, Dai JR. Diagnosis and differential diagnosis of MRI in hypertensive encephalopathy. Chinese Journal of Magnetic Resonance Imaging 2014;5:15-8.

35. Granberg T, Bergendal G, Shams S, Aspelin P, KristoffersenWiberg M, Fredrikson S, Martola J. MRI-Defined Corpus Callosal Atrophy in Multiple Sclerosis: A Comparison of Volumetric Measurements, Corpus Callosum Area and Index. J Neuroimaging 2015;25:996-1001.

36. Tsivgoulis G, Katsanos AH, Grigoriadis N, Hadjigeorgiou GM, Heliopoulos I, Papathanasopoulos P, Dardiotis E, Kilidireas C, Voumvourakis K; HELANI (Hellenic Academy of Neuroimmunology). The effect of diseasemodifying therapies on brain atrophy in patients with clinically isolated syndrome: a systematic review and metaanalysis. Ther Adv Neurol Disord 2015;8:193-202.

37. Xu Z, Xiao N, Chen Y, Huang H, Marshall C, Gao J, Cai Z, Wu T, Hu G, Xiao M. Deletion of aquaporin-4 in APP/ PS1 mice exacerbates brain A $\beta$ accumulation and memory deficits. Mol Neurodegener 2015;10:58.

38. Chakraborty S, ThimmaReddygari J, Selvaraj D. G-Lymphatic, Vascular and Immune Pathways for A $\beta$ Clearance Cascade and Therapeutic Targets For

Cite this article as: Liu XY, Ma GY, Wang S, Gao Q, Guo C, Wei Q, Zhou X, Chen LP. Perivascular space is associated with brain atrophy in patients with multiple sclerosis. Quant Imaging Med Surg 2022;12(2):1004-1019. doi: 10.21037/qims-21-705
Alzheimer's Disease. Comb Chem High Throughput Screen 2021;24:1083-92.

39. Andravizou A, Dardiotis E, Artemiadis A, Sokratous M, Siokas V, Tsouris Z, Aloizou AM, Nikolaidis I, Bakirtzis C, Tsivgoulis G, Deretzi G, Grigoriadis N, Bogdanos DP, Hadjigeorgiou GM. Brain atrophy in multiple sclerosis: mechanisms, clinical relevance and treatment options. Auto Immun Highlights 2019;10:7.

40. Trapp BD, Vignos M, Dudman J, Chang A, Fisher E, Staugaitis SM, Battapady H, Mork S, Ontaneda D, Jones SE, Fox RJ, Chen J, Nakamura K, Rudick RA. Cortical neuronal densities and cerebral white matter demyelination in multiple sclerosis: a retrospective study. Lancet Neurol 2018;17:870-84.

41. Stephenson E, Nathoo N, Mahjoub Y, Dunn JF, Yong VW. Iron in multiple sclerosis: roles in neurodegeneration and repair. Nat Rev Neurol 2014;10:459-68.

42. Bocheng W, Chaofeng L, Chuan C, Haiyong H, Tengchao H, Qun G, Ying G. Intracranial lymphatic drainage discharges iron from the ventricles and reduce the occurrence of chronic hydrocephalus after intraventricular hemorrhage in rats. Int J Neurosci 2020;130:130-5.

43. Zhou W, Shen B, Shen WQ, Chen H, Zheng YF, Fei JJ. Dysfunction of the Glymphatic System Might Be Related to Iron Deposition in the Normal Aging Brain. Front Aging Neurosci 2020;12:559603. 